\title{
XERIC climate-control system for energy-efficient electric vehicles: first experimental results and numerical evaluation of the overall performance
}

\author{
Carlo Isetti ${ }^{(1)}$, Enrico Nannei ${ }^{(3)}$ \\ TICASS, Innovative Technologies for \\ Environmental Control and Sustainable \\ Development, Via D. Fiasella, 3/16 Genoa, \\ Italy \\ Email: isetti@leonardo.arch.unige.it \\ e.nannei@gmail.com
}

\author{
Stefano Lazzari ${ }^{(2)}$ \\ DAD, Department of Architecture \\ and Design, University of Genoa, \\ Stradone S. Agostino, 37 -16123 \\ Genoa, Italy \\ Email: stefano.lazzari@unige.it
}

\author{
Bernardo Cerrai ${ }^{(4)}$, Sergio Nari ${ }^{(5)}$ \\ FRIGOMAR, Marine Refrigeration and \\ Air Conditioning Systems, Via V. \\ Veneto, 16042 Carasco, Genoa, Italy \\ Email: b.cerrai@frigomar.com \\ s.nari@frigomar.com
}

\begin{abstract}
The paper reports the main developments and results achieved so far within the EU-funded $\mathbf{H 2 0 2 0}$ XERIC project, which is aimed at developing a new climate control system that is able to increase Battery Electric Vehicles (BEVs) autonomy thanks to its high energy efficiency. The XERIC system combines a traditional Vapor Compression Cycle (VCC) with a Liquid Desiccant Cycle (LDC), by taking advantage of an innovative component, called Three-Fluids Combined Membrane Contactor (3F-CMC). The two experimental setups arranged to test $\mathrm{LDC}$ alone and the overall XERIC system are presented. Then, the experimental results obtained in the first test campaign are given and discussed. Finally, the numerical tool developed in the Matlab/Simulink environment for the evaluation of the performance of the overall XERIC system is introduced and a preliminary comparison between numerical results and experimental measurements is provided.
\end{abstract}

Keywords- XERIC project; Hybrid systems for air conditioning; liquid desiccant cycle; innovative membrane contactors; energy-efficient electric vehicles; AC mobile system.

\section{INTRODUCTION}

According to the recent trends in scientific research, automotive market leanings and grants by European Commission, a wide diffusion of Battery Electric Vehicles (BEVs) is highly investigated and promoted, since it can noteworthy help improving energy savings and environmental sustainability. However, some major drawbacks are delaying the market penetration of BEVs against traditional Internal Combustion Engine Vehicles (ICEVs). The main issues that have to be faced are the limited driving range of BEVs, the small availability of charging stations, the long time needed for a full charge and the high purchase cost compared to ICEVs.
Among the first calls within the Horizon 2020 EU program, one was specifically devoted to face and solve the issue represented by the limited autonomy range of BEVs by means of, but not limited to, innovative approaches to the onboard thermal management system. The EU project "XERIC" (Innovative Climate-Control System to Extend Range of Electric Vehicles and Improve Comfort) is one of the three $\mathrm{H} 2020$ funded projects within the "Green Vehicles" call on optimized and systematic energy management in electric vehicles. The development of a small-scale prototype of an energy-saving climate control system for EVs currently on the market is the main aim of the XERIC project. Indeed, as known, one of the most energy-demanding system on board is represented by the air-conditioning system, which can absorb up to 40$60 \%$ of the available energy in summer conditions. In detail, XERIC system will:

1. reduce more than $50 \%$ the energy used all over the year for heating, cooling and dehumidifying air compared to existing systems;

2. reduce more than $30 \%$ the energy used for air cooling/dehumidifying in extreme summer conditions (i.e., external air at $\mathrm{T}=30{ }^{\circ} \mathrm{C}$ and $\mathrm{RH}=60 \%$ ) to guarantee comfort in the passenger cabin (i.e., $\mathrm{T} \approx 25{ }^{\circ} \mathrm{C}$ and $\mathrm{RH} \approx 50 \%$;

3. guarantee the after-project easy industrial scale-up and the customization of system;

4. guarantee an adequate working life;

5. withstand the different external air temperature ranges across Europe;

6 . profitably use the components currently installed in EVs;

7. guarantee a reasonable cost (to OEM), which depends mainly on car size, when produced at industrial level.

The ambitious goal reported above is pursued by the XERIC system by avoiding the waste of energy imposed by a traditional Vapour Compression Cycle (VCC) in 
order to dehumidify air. Indeed, air dehumidification in this cycle is obtained by first cooling the air to the dew point temperature and then by undercooling it until the desired specific humidity is reached [1]. XERIC system is a hybrid system in which air can be dehumidified without the need to be cooled below the dew-point thanks to the combination of a Liquid Desiccant Cycle (LDC, which deals mainly with the latent load) with a traditional VCC (which faces the sensible load) [2,3].

In the present paper, the two experimental setups arranged to test the Liquid Desiccant Cycle and the overall XERIC system are presented together with the main technological advancements involved. Then, the experimental results obtained in the first test campaign are shown and discussed. Finally, the numerical tool developed in the Matlab/Simulink environment for the evaluation of the performance of the overall XERIC system is introduced and a preliminary comparison between numerical results and experimental measurements is given.

\section{LIQUID DESICCANT CYCLE}

The XERIC system combines a traditional Vapor Compression Cycle (VCC) with a Liquid Desiccant Cycle (LDC), by taking advantage of an innovative component, called Three-Fluids Combined Membrane Contactor (3FCMC) $[4,5]$. This component is an innovative heat and mass exchanger, which works simultaneously with three fluids: air, liquid desiccant and refrigerant. The vapour present in moist air can be removed (absorbed) by the liquid desiccant through a semi-permeable membrane, which can be crossed by vapour but not by liquid, thus allowing a physical separation between air and desiccant. Figure 1a shows the 3D CAE design of the $3 \mathrm{~F}-\mathrm{CMC}$ and Figure $1 \mathrm{~b}$ the small-scale prototype of it, i.e. a prototype that is able to treat about one third of the air flow rate that is conveyed to the cabin for a $\mathrm{C}$-segment car. Details about the patented 3F-CMC can be found in [4].

As sketched in Figure 2, the architecture of the XERIC system is obtained by simply adding the few components constituting the LDC to the usual components of a VCC. In the XERIC system [5] there are two 3F-CMCs: the first one, 3F-CMC1, dehumidifies and partially cools the process air, while the second one, $3 \mathrm{~F}-\mathrm{CMC} 2$, acts as a regenerator to re-concentrate the desiccant diluted solution exiting the 3F-CMC1. An intermediate heat exchanger (HE), operating between the cold and the warm solutions, reduces the parasitic heat transfer enhancing the performance of the LDC. Lastly, a small pump is needed to move the desiccant solution within the closed and sealed loop between 3F-CMC1 and 3F-CMC2.

The $3 \mathrm{~F}-\mathrm{CMC} 1$, set on the renewal air path, handles this air flow before it mixes with the recirculated air flow from the cabin (see the recycle door set before the fan, in Fig.2).
The air flow through 3F-CMC1 can be excluded by a second door when the desiccant cycle does not work. As shown in the sketch, downstream the fan the air flow can be partially or totally conveyed to the heat exchanger HE1 through a third door, whereas further doors may address the air flow to cabin vent, to windscreen defrost and to cabin floor.

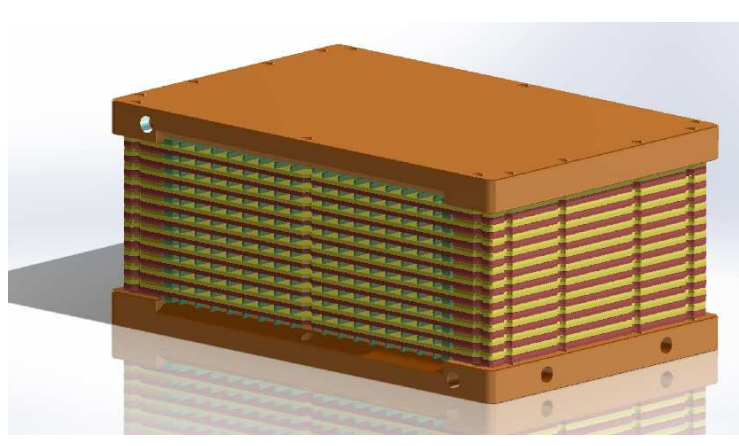

Fig.1a-CAE design of the 3F-CMC.

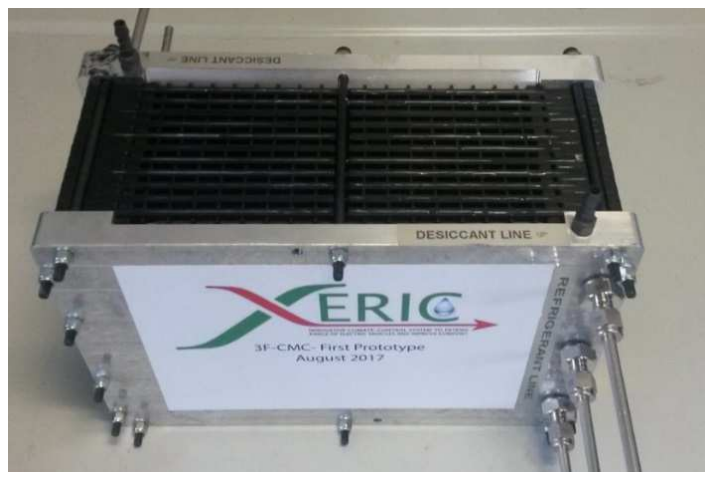

Fig. $1 \mathrm{~b}$ - The small-scale prototype of the 3F-CMC.

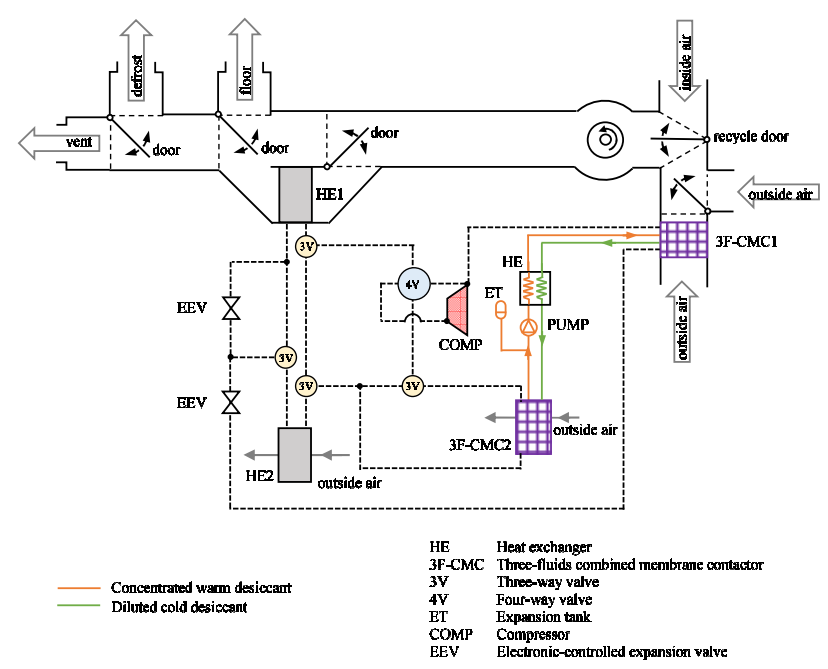

Fig. 2 - The XERIC system architecture.

The further presence of a 4-ways valve permits the system to act as a heat pump in wintertime, thus allowing a noteworthy energy-saving with respect to direct electric 
heating. Moreover, some 3-ways valves allow the system to fulfill different tasks, as described in [5]: cooling \& dehumidification, dehumidification only, heating \& dehumidification and heating only.

The performance of the LDC in terms of water vapour absorption and desorption from/to air have been investigated, both experimentally and numerically. Figure 3 shows the scheme of the experimental setup realized for these tests campaign in the TICASS laboratory.

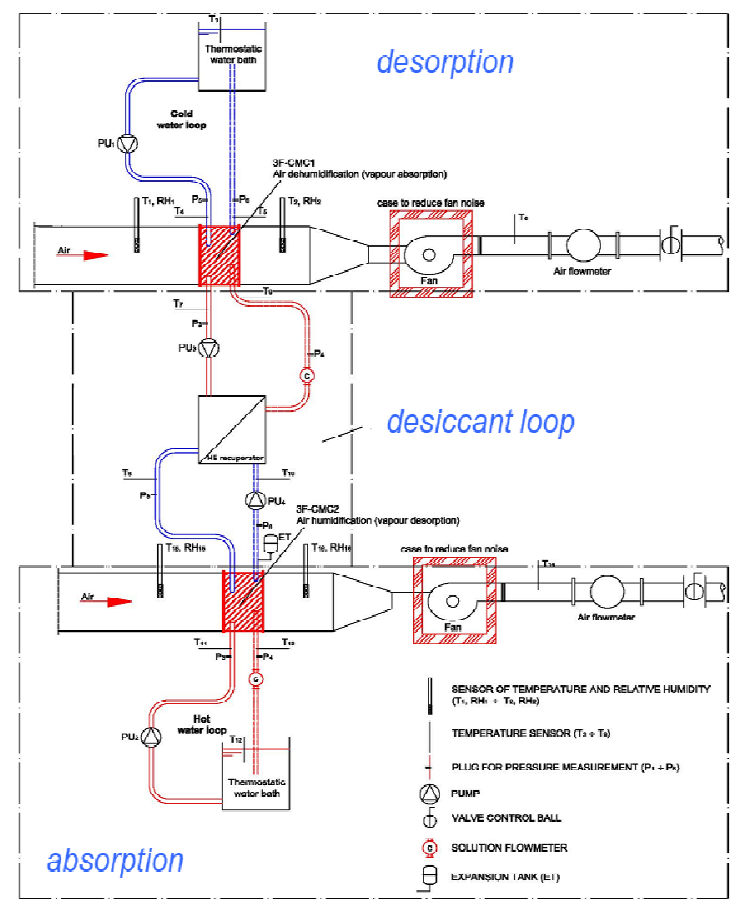

Fig.3 - Conceptual scheme of the experimental setup for testing LDC performance.

Since the aim of these experimental tests was mainly evaluating the performance of the 3F-CMCs and the LDC, the refrigerant was replaced by a high flow rate of chilled water through 3F-CMC1 (warm water in 3F-CMC2), produced by means of thermostatic water baths. The adopted desiccant is Lithium Chloride $(\mathrm{LiCl})$ aqueous solution. A titanium coaxial heat exchanger allows a heat exchange between the cold and the warm $\mathrm{LiCl}$ solutions. Different external air conditions in terms of both temperature and relative humidity were considered by acting on an inlet air heater made by electric resistances and a specifically designed vapour inlet control. Figure 4 shows the actual experimental setup configuration, together with the pc devoted to data acquisition and elaboration. The desiccant flow rate, measured by digital flowmeter, is circulated by a Flui-o-Tech® gear micropump.

One of the first test made was about the air pressure drop across the 3F-CMC. Indeed, in order to profitably use the components currently installed in EVs, and the current fan in particular, the design aimed at containing the pressure drop below $100 \mathrm{~Pa}$. Figure 5 shows the result obtained when an air flow rate of $67 \mathrm{~m}^{3} / \mathrm{h}$ is considered. Since the total air flow rate to the cabin is about $300-400$ $\mathrm{m}^{3} / \mathrm{h}$, and $100-200 \mathrm{~m}^{3} / \mathrm{h}$ is recirculated from the cabin, the full 3F-CMC treats about $200 \mathrm{~m}^{3} / \mathrm{h}$. Thus a small-scale prototype, which is one third of the full size, treats about $67 \mathrm{~m}^{3} / \mathrm{h}$ of renewal air. The measured pressure drop, 86 $\mathrm{Pa}$, is lower than the limit, confirming a proper design of the component.

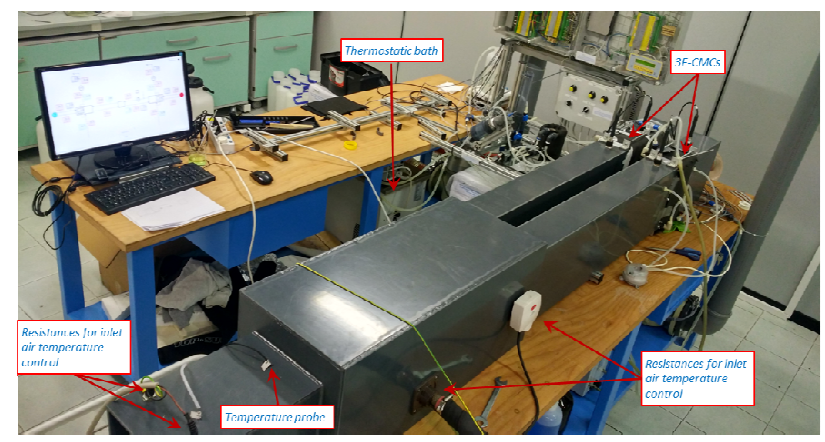

Fig.4 - Experimental setup for testing LDC.

The experimental campaign consisted in several tests on both absorption and desorption performance carried out by measuring air temperature and R.H., upstream and downstream the 3F-CMCs. Before the tests, all sensors were carefully calibrated against fixed points of relative humidity obtained with LiCL and $\mathrm{NaCl}$ saturated solutions [6].

As an example, Figure 5 shows the vapour absorption obtained by $3 \mathrm{~F}-\mathrm{CMC1}$. In the tested conditions, more than $500 \mathrm{~g} / \mathrm{h}$ of vapour is removed from air by 3F-CMC1 for square meter of membrane, with a dependence on the liquid desiccant concentration adopted.

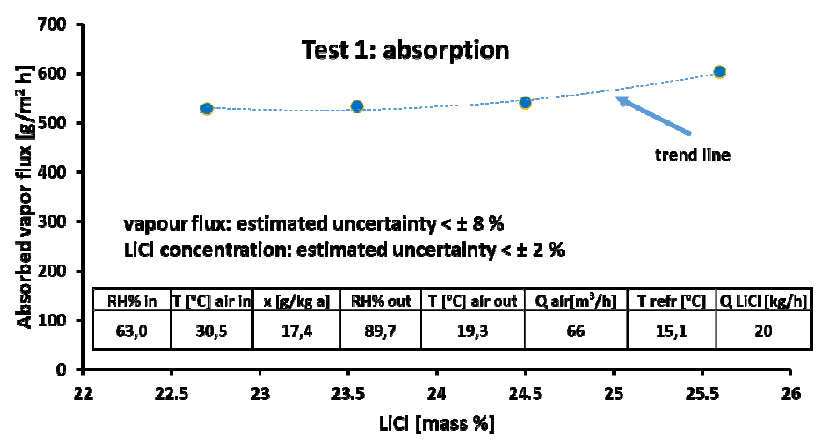

Fig.5 - Vapour absorption test for warm and moist air inlet conditions.

Figure 6 shows the time trend of the $\mathrm{LiCl}$ concentration for the full LDC under given test conditions: a stabilized equilibrium value is finally reached, confirming that 3FCMC2 is properly re-generating the diluted desiccant solution coming from $3 \mathrm{~F}-\mathrm{CMCl}$. The $\mathrm{LiCl}$ concentration 
was measured by probes sensible to the electric conductivity of the solution (BC Electronics ST3214).

LCl concentration vs time

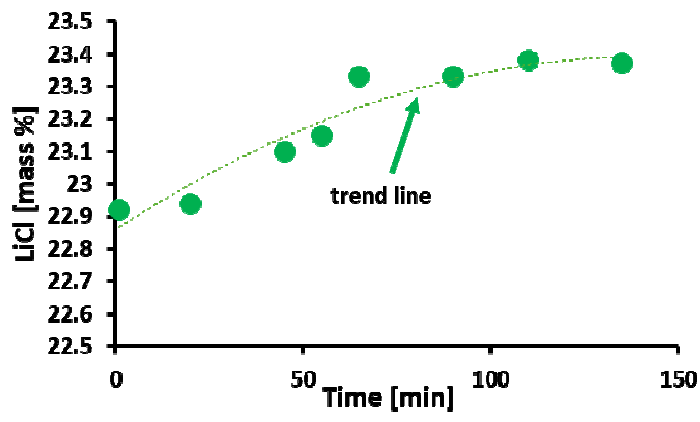

Fig.6 - Time trend of the desiccant concentration.

\section{XERIC SYSTEM}

Besides the tests on LDC, the overall XERIC system is undergoing a campaign of experimental tests in climatic chamber to determine the performance in comparison to a traditional AC system for BEV set as reference. In detail, the Reference Climate-Control System (RCCS) employs a VCC to cool and to dehumidify the air in summer/intermediate seasons while performs the heating action in winter by means of an electric heater. The conceptual scheme of the experimental setup for testing the XERIC system is shown in Figure 7.

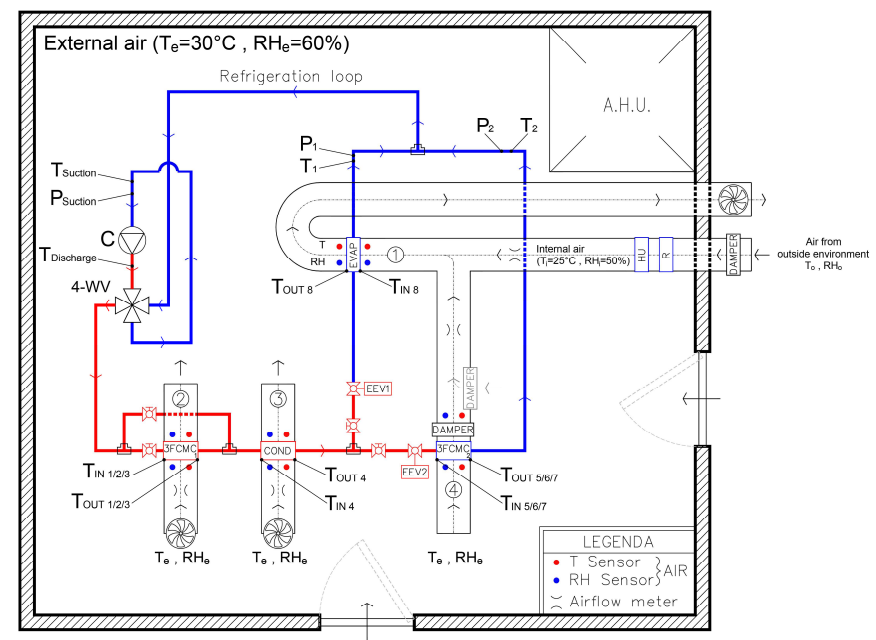

Fig. 7 - Conceptual scheme of the experimental setup for testing XERIC system performance.

The refrigerant circuit used for XERIC system is slightly different from a standard VCC because two electronic expansion valves set in parallel are used: one controls the refrigerant flow rate towards the evaporator, the other one towards the 3F-CMCs. The tuning of the electronic control system that operates the valves is now in progress.
Figure 8 shows the overall XERIC system, which is composed by the VCC (items 1/2/3) and by the LDC (items 4/5/6/7). Desiccant solution is flowing inside the circuit by means of a dedicated gear pump (7) and a recuperator (6) allows to control the parasitic heat coming from the two 3F-CMCs (4/5). The condenser (2) and the evaporator (3) of the VCC are taken from a C-segment electric car and their frontal area have been reduced to $1 / 3$, according to the adoption of small-scale prototypes of $3 \mathrm{~F}$ CMCs. The other components (1) of the VCC are the compressor (rotary type, moved by a BLDC motor) and the inverter system controlling the BLDC motor (made of two main components, the power module and the control module).

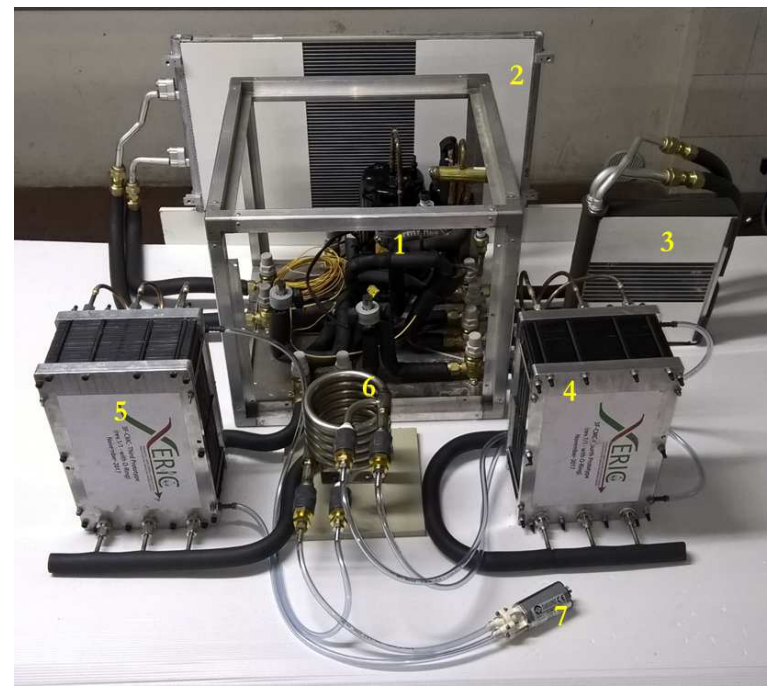

Fig. 8 - XERIC system components.

The experimental setup prepared in the FRIGOMAR laboratory allows the management of two independent ambient conditions in one single room, one simulating the cabin conditions, the other one recreating the outdoor ambient conditions. The outdoor thermal hygrometric conditions are obtained in the climatic chamber by using an air handling unit (AHU) and a heating resistance humidifier. The indoor cabin thermal hygrometric conditions are obtained by means of a dedicated ducted AHU.

The aim of these preliminary tests is to check the proper control of extreme summer conditions (outside temperature $30^{\circ} \mathrm{C}$, outside relative humidity $60 \%$ ) and simultaneously to verify the proper working of XERIC system. Please note that further tests about the performance of the XERIC system are in progress and the results will be the topic of an upcoming paper. The preliminary results show that the desiccant circuit is stable, as proved by constant concentration and temperature of the desiccant, which means that the vapor mass flow rate absorbed during the dehumidification process is equal to the vapor mass flow rate desorbed during the 
concentration process. Moreover, the EEVs control the refrigerant flow rates towards the evaporator and the $3 \mathrm{~F}$ CMCs properly, since the compressor inlet temperature remains constant.

\section{NUMERICAL TOOL}

As a parallel activity to the experimental study, a numerical tool that can simulate the XERIC system functioning and forecast its energy performance has been developed. The software has been implemented in the Matlab/Simulink environment (@Mathworks), since it can easily handle complex systems made by several components, as is the case for XERIC system. It is seen as a succession of separated models, each being a standalone code, which are able to exchange information to/from the other connected models. Each Simulink block constituting the model of a component can be easily connected to simulate more complex systems. Every block needs input variables and delivers outlet variables; output and inlet variables are linked together by means of the governing equations describing the physical behavior of the component itself. Each component (3F-CMCs, heat exchanger, evaporator, condenser, compressor, expansion valves, etc.) has been modeled alone under the following main simplifying assumptions: steady-state regime; unidirectional and not compressible air flows; negligible heat transfer with the external environment; absence of uneven flow distributions within the components. The main heat and mass transfer coefficients needed in the 1D model of the 3F-CMC (e.g., air side heat transfer coefficient) have been obtained by $3 \mathrm{D}$ accurate numerical simulations made by means of commercial CFD software packages (Fluent by Ansys and Comsol Multiphysics by COMSOL) or determined through theoretical analyses [7, 8]. The Simulink models of evaporator, condenser and 3F$\mathrm{CMC}$ can work relying on expressions taken from the literature [9] for determining fluid properties as well as on the experimental data enclosed in the REFPROP 9 software by NIST [10].

Figure 9 shows the Simulink blocks simulating the 3FCMCs: Figure 9a refers to the 3F-CMC1 in absorption, while Figure $9 \mathrm{~b}$ to the $3 \mathrm{~F}-\mathrm{CMC} 2$ in desorption.

The overall Simulink scheme of the XERIC system is depicted in Figure 10. The big red block at the center of the figure contains several other linked blocks, which simulate the VCC and the LDC. Moreover, the gray block evaluates the real cabin heat and mass balance and determines the Predicted Mean Vote (PMV) and the Predicted Percentage of Dissatisfied (PPD) according to Fanger's theory [11] in order to account for the thermal comfort inside the cabin. As known, the goal is to obtain a PPD as close as possible to $5 \%$, as shown in the figure $(5.8 \%)$

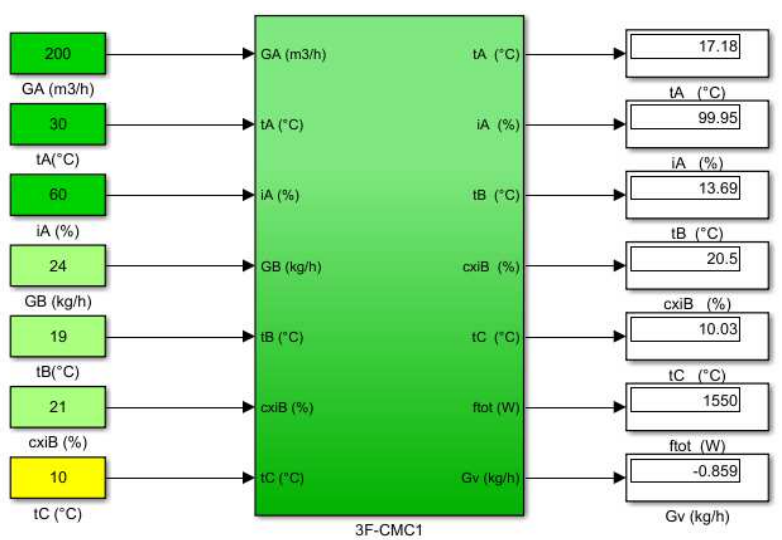

Fig.9a - Simulink block of the 3F-CMC1, with input data (left) and output data (right).

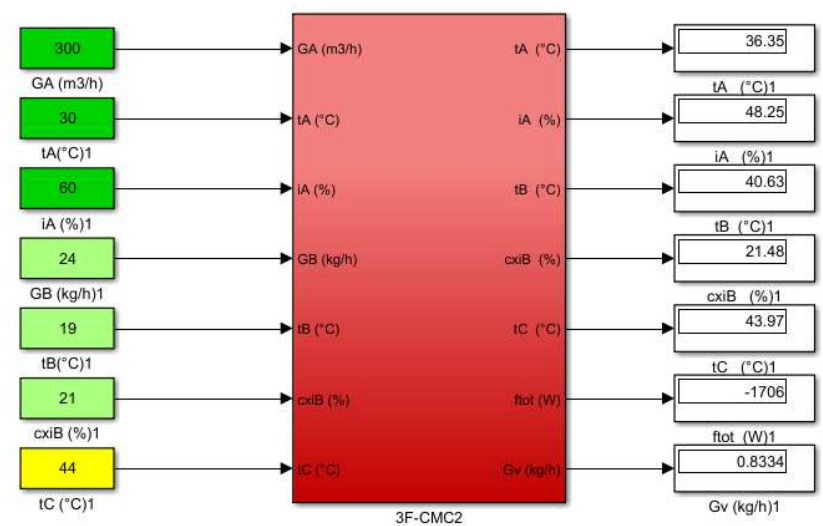

Fig.9b - Simulink block of the 3F-CMC2, with input data (left) and output data (right).

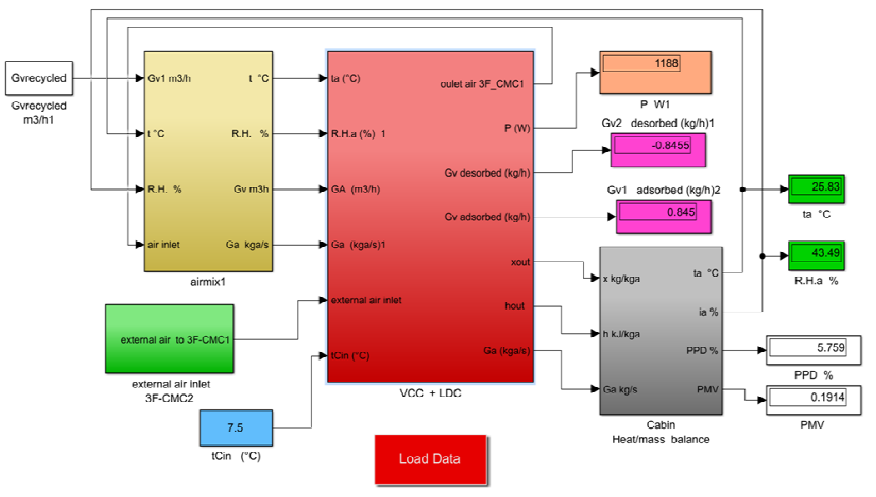

Fig.10 - Simulink scheme of the XERIC system.

The numerical tool has been used also to evaluate the absorption/desorption performance of the 3F-CMCs. Figure 11 shows the experimental results (points) together with the numerical ones (line) for an absorption test. 


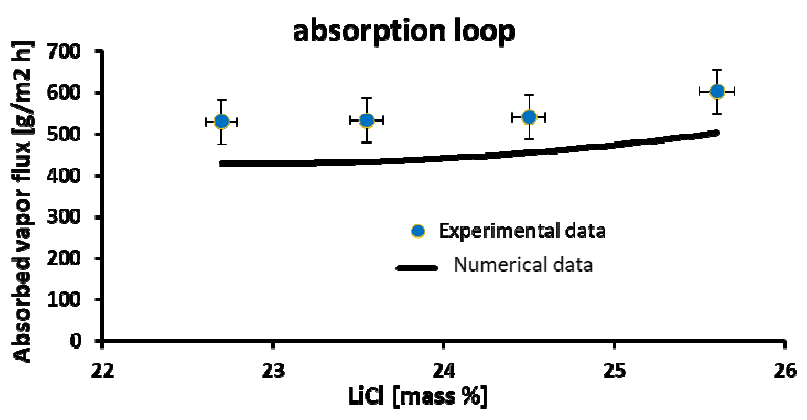

Fig.11 - Comparison between experimental and numerical results for an absorption test.

As can be inferred from the figure, the numerical results are in good agreement with experimental ones: the predicted trend is the same, whereas the predicted values are slightly underestimated, which can be easily due to the values of the membrane properties adopted in the model. Studies are under progress to identify this difference and then allow the fine-tuning of the numerical model.

\section{CONCLUSION}

In the present paper, the two experimental setups developed to test the innovative XERIC climate control system for enhancing driving range of Battery Electric Vehicles are presented. In detail, the setups refer to the Liquid Desiccant Cycle and the overall system, respectively. The numerical tool developed in order to predict the performance of each component of the system and the system itself is also presented. The results of the first experimental campaign are shown and compared to the numerical data. The experimental results confirm the expected performance of the XERIC system and the comparison with the numerical predictions substantially validates the numerical tool. Further tests are in progress and will be presented in a next paper.

\section{ACKNOWLEDGMENT}

This work is part of a project that has received funding from the European Union's Horizon 2020 research and innovation programme under grant agreement $\mathrm{N}^{\circ} 653605$.

\section{REFERENCES}

[1] ASHRAE Handbook, Fundamentals, SI Edition, 2005

[2] P. Mazzei, F. Minichiello, D. Palma, HVAC dehumidification systems for thermal comfort: a critical review, Applied Thermal Engineering, Vol. 25, pp. 677-707, 2005.

[3] L. Mei, Y.J. Dai, A technical review on use of liquid-desiccant dehumidification for air-conditioning application, Renewable \& Sustainable Energy Reviews, Vol.12, pp. 662-689, 2008.

[4] C. Isetti, S. Lazzari, E. Nannei, T. Prill, O. Iliev, S. Hariri, New Climate-Control Units for More Energy-Efficient Electric Vehicles: the Innovative Three-Fluids Combined Membrane Contactor, in the Proc. of EVER2017 Conference, pp. 1-5, Monaco, April 2017.

[5] C. Isetti, S. Lazzari, E. Nannei, B. Cerrai, S. Nari, New ClimateControl Units for More Energy-Efficient Electric Vehicles: system architecture, in the Proc. of EVER2017 Conference, pp. 1-7, Monaco, April 2017.

[6] L. Greenspan, Humidity fixed points of binary saturated aqueos solutions, Journal of Resarch of National Bureau of Standards, 81A(1), 1977.

[7] J.R. Welty, C.E. Wicks, R. Wilson, Fundamentals of Momentum, Heat and Mass Transfer, J.Wiley \& Sons, N.Y., 1969.

[8] F.P. Incropera, D.P. Dewitt, Fundamentals of Heat Transfer, John Wiley and Sons, Inc. 1985.

[9] A.C. Cleland, Polynomial curve-fits for refrigerant thermodynamic properties: extension to include R134a, Rev. Int. Froid, 17, n⿳⺈ 4, pp. 245,249, 1992.

[10] REFPROP, NIST Standard reference database, Version 9

[11] UNI-EN ISO 7730: "Ergonomics of thermal environments: analytical determination and interpretation of thermal comfort using calculation of the PMV and PPD indices and local thermal comfort criteria”, 2005 